\title{
Low-pressure pulmonary recruitment maneuver: equal to or worse than moderate-pressure pulmonary recruitment maneuver in preventing postlaparoscopic shoulder pain? A randomized controlled trial of 72 patients
}

\author{
Gulseren Yilmaz ${ }^{1}$, Huseyin Kiyak², Aysu Akca², Ziya Salihoglu \\ ${ }^{1}$ Department of Anesthesiology and Reanimation, University of Health Sciences, Faculty of Medicine, Kanuni Sultan Suleyman \\ Hospital, Istanbul, Turkey \\ 2Department of Obstetrics and Gynecology, University of Health Sciences, Faculty of Medicine, Kanuni Sultan Suleyman Hospital, \\ Istanbul, Turkey \\ ${ }^{3}$ Department of Anesthesiology and Reanimation, Istanbul University, Cerrahpasa Medical School, Istanbul, Turkey
}

Videosurgery Miniinv 2020; 15 (3): 519-525

DOI: https://doi.org/10.5114/wiitm.2019.89831

\begin{abstract}
Introduction: The pulmonary recruitment maneuver (PRM) has emerged as an effective way of reducing post-laparoscopic shoulder pain (PLSP). However, the optimal lower pressure level for a PRM to reduce PLSP has not yet been investigated.

Aim: To compare the efficacy of the low-pressure PRM with moderate-pressure PRM in preventing PLSP. Material and methods: Seventy-two ASA I-II patients who were scheduled for gynecologic LS for non-malignant conditions were enrolled in this study. Group 1 included patients who received the PRM at a maximum pressure of 30-40 cm $\mathrm{H}_{2} \mathrm{O}$ in a semi-Fowler position and group 2 included patients who received the PRM at a maximum pressure of $15 \mathrm{~cm}$ $\mathrm{H}_{2} \mathrm{O}$ in a semi-Fowler position. The primary outcome of the study was the difference in PLSP between the two groups. Results: There were no significant differences in PLSP and wound pain VAS scores between patients receiving the $P R M$ at $30 \mathrm{~cm} \mathrm{H}_{2} \mathrm{O}$ and $15 \mathrm{~cm} \mathrm{H}_{2} \mathrm{O}$ during postoperative pain monitoring $(p<0.05)$. The groups were also similar with respect to ambulation time $(p=0.215)$, length of hospital stay $(p=0.556)$ and the height of the pneumoperitoneum measured on chest $X$-ray $(p=0.151)$.

Conclusions: The low-pressure PRM (15 $\mathrm{Cm} \mathrm{H}_{2} \mathrm{O}$ pressure) provides similar efficacy as the moderate-pressure PRM (30-40 $\mathrm{cm} \mathrm{H}_{2} \mathrm{O}$ ) in terms of PLSP, wound pain, height of pneumoperitoneum, time of ambulation and length of hospital stay. We suggest that lower maximal inspiratory pressure of $15 \mathrm{~cm} \mathrm{H}_{2} \mathrm{O}$ might be preferred to avoid the potential complications of the PRM with higher pressures.
\end{abstract}

Key words: laparoscopy, pulmonary recruitment maneuver, low pressure, shoulder pain, pneumoperitoneum.

\section{Introduction}

The trend in surgery has shifted from invasive procedures to minimally invasive procedures with the introduction of laparoscopic surgery (LS) in 1987. LS has provided many advantages including rapid recovery, shorter hospital stay, and less postoperative pain compared to conventional open surgery.

\section{Address for correspondence}

Gulseren Yilmaz MD, Department of Anesthesiology and Reanimation, University of Health Sciences, Faculty of Medicine,

Kanuni Sultan Suleyman Hospital, Istanbul, Turkey, e-mail: drgulseren83@gmail.com 
Although LS is associated with a reduction in wound pain as a consequence of the smaller incisions, new issues that the surgeons and the anesthetists were not familiar arose with wide implementation of LS.

Post-laparoscopic shoulder pain (PLSP) is predominantly attributed to the accumulation of carbon dioxide below the diaphragm and to the resultant irritation of the phrenic nerve caused by the diaphragmatic stretching. The reported prevalence of PLSP has reached $80 \%$ in some series [1]. Therefore, reducing the PLSP to improve patients' wellbeing has been one of the main objectives of the surgical team dealing with LS. However, the vast majority of these techniques demonstrated controversial outcomes and were time-consuming and impractical for use in daily practice. The pulmonary recruitment maneuver (PRM) has emerged as an effective way of reducing PLSP through increasing the intraperitoneal pressure and assisting the evacuation of the remaining carbon dioxide. In a recent trial from our institute, it was shown that the PRM with 30 to $40 \mathrm{~cm} \mathrm{H}_{2} \mathrm{O}$ pressure in a semi-Fowler position ( $30^{\circ}$ head-of-bed elevation) leads to a significant reduction in PLSP compared to the PRM in the neutral position or compared to the passive evacuation of the abdominal carbon dioxide without the PRM [2]. However, the PRM with higher pressure carries the risk of pulmonary barotrauma complications and hemodynamic deterioration [3, 4]. In addition, whether a low-pressure PRM could provide similar efficacy as the traditional moderate-pressure PRM has not yet been investigated.

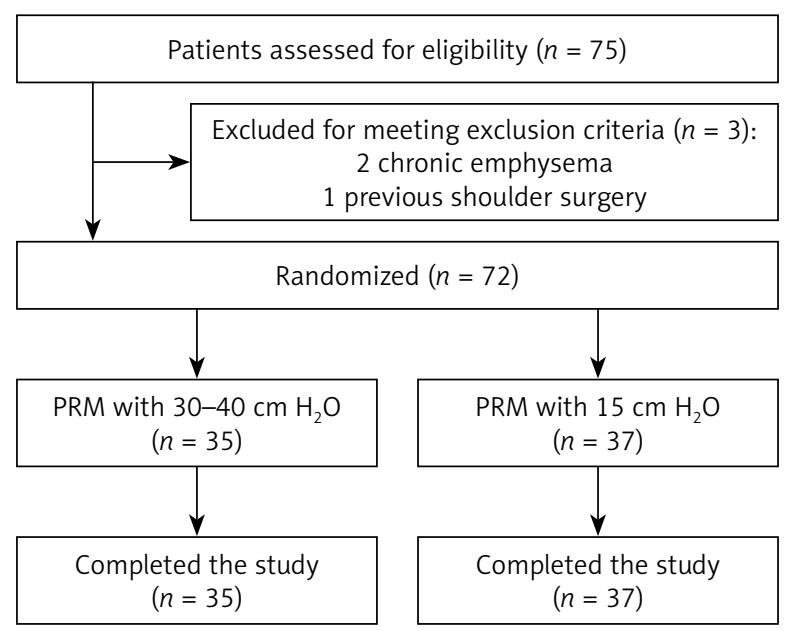

Figure 1. Flow diagram demonstrating patient allocation
We hypothesized that the low-pressure PRM may confer a similar benefit as the moderate-pressure PRM in terms of shoulder pain, wound pain, height of pneumoperitoneum, time of ambulation and length of hospital stay.

\section{Aim}

The present randomized, controlled, double-blind study was designated for a particular purpose: to compare the efficacy of the low-pressure PRM with the moderate-pressure PRM in preventing PLSP. For this purpose, patients undergoing LS were allocated to the PRM with either $15 \mathrm{~cm} \mathrm{H}_{2} \mathrm{O}$ pressure or $30-40 \mathrm{~cm} \mathrm{H}_{2} \mathrm{O}$ pressure and their impact on PLSP was compared.

\section{Material and methods}

\section{Patient selection}

The present randomized, double-blind, prospective study enrolled 72 ASA I-II patients aged between 18 and 70 years who were scheduled for gynecologic LS for non-malignant conditions in Istanbul Health Sciences University, Kanuni Sultan Suleyman Education and Research Hospital between July 2019 and August 2019. Exclusion criteria were as follows: chronic shoulder or epigastric pain, previous lung or shoulder surgery; chronic emphysema; pneumothorax; pregnancy. Patients who were potential candidates for concomitant upper abdominal surgery and those unable to express active pain were also excluded (Figure 1). The study protocol was approved by the Institutional Ethics Committee (KAEK 2019/194) and registered at ClinicalTrials.gov (NCT03970473). The study was performed in accordance with the most recent version of the Helsinki Declaration. Power calculations based on our pilot study with 20 patients revealed that (visual analogue scale score for PLSP at postoperative $6^{\text {th }} \mathrm{h}$ in group 1: $6.8 \pm 1.2$ vs. group 2: $5.7 \pm 0.9$, an effect size of 1.00, $\alpha$ error: 0.05 and power: 0.95 ) at least totally 42 patients would be required for an adequate sample size [5].

\section{Anesthesia and surgery}

All patients received a standardized general anesthesia procedure. Premedication was performed using $0.03 \mathrm{mg} / \mathrm{kg}$ of midazolam intravenously. Intravenous propofol $2 \mathrm{mg} / \mathrm{kg}$, fentanyl $1 \mathrm{mg} / \mathrm{kg}$ and $0.8 \mathrm{mg} / \mathrm{kg}$ rocuronium were utilized to induce gen- 
eral anesthesia which was further maintained with sevoflurane 2-3 vol\%. Ventilation was performed in a volume-controlled mode at a tidal volume of $7-9 \mathrm{ml} / \mathrm{kg}$. None of the patients received positive end-expiratory pressure (PEEP). The level of neuromuscular block was measured by acceleromyography (TOF-Watch-SX Monitor, Organon Teknika, Dublin, Ireland). Train-of-four (TOF) was monitored throughout the operation and an additional rocuronium bolus of $0.15 \mathrm{mg} / \mathrm{kg}$ was administered when the TOF value was $25 \%$.

An experienced LS team performed all LS procedures. All patients underwent a multiport LS comprising a 12-mm optical port placed at the umbilicus, 5 - $\mathrm{mm}$ additional operating ports placed in the lower left and right quadrants and a 5-mm suprapubic port. Strict attention was paid to maintaining the intra-abdominal pressure about $12 \mathrm{~mm} \mathrm{Hg}$ during the procedure. All patients were placed in the $15-20^{\circ}$ Trendelenburg position. Following the surgery, the lateral ports were removed and the intra-abdominal gas was removed via the main umbilical port. A sealed envelope identifying the patients' group was then opened by a research fellow.

Using random allocation software (www.randomization.com), participants were assigned to one of two intervention groups in a $1: 1$ ratio with simple randomization: group 1 included patients who received the PRM which consisted of five manual pulmonary inflations where each positive pressure inflation was done for $5 \mathrm{~s}$ at a maximum pressure of 30 to $40 \mathrm{~cm} \mathrm{H} \mathrm{H}_{2} \mathrm{O}$ in a semi-Fowler position ( $30^{\circ}$ headof-bed elevation), and group 2 included patients who received the PRM at a maximum pressure of $15 \mathrm{~cm} \mathrm{H}_{2} \mathrm{O}$ in a semi-Fowler position. The random allocation sequence was generated by a research fellow. Following the completion of the surgery a sealed envelope indicating the group of the index patient was then opened by the anesthesiologist managing the anesthesia procedure. All patients were positioned in $30^{\circ}$ head-of-bed elevation (semi-Fowler position) before the PRM and patients' position was maintained until the PRM was completed. Group 1 patients received the PRM at a pressure of 30 to $40 \mathrm{~cm} \mathrm{H}_{2} \mathrm{O}$ and group 2 patients received the PRM at a pressure of $15 \mathrm{~cm} \mathrm{H}_{2} \mathrm{O}$ and the main port was then removed. PRM intervention was also performed by the anesthesiologist managing the anesthesia procedure. Participants and the research staff assessing the outcomes were blinded to patient data.

\section{Primary and secondary outcomes}

The primary outcome of the study was the difference in PLSP between the two groups which was recorded at postoperative 6,12 and $24 \mathrm{~h}$ by a research fellow who was blinded to patients' groups. A visual analogue scale (VAS) based on a 0-10 scale, with 0 meaning no pain and 10 the most intense pain ever experienced, was used for grading PLSP and wound pain. In the case of any pain VAS $>4$ dexketoprofen trometamol (50 mg i.v.) was administered as a rescue analgesic. Upright posteroanterior (PA) chest X-ray imaging was performed in all patients at the postoperative $24^{\text {th }} \mathrm{h}$. The height of the gas bubble under each hemi-diaphragm was measured and their sums were divided into two to estimate the residual gas volume (Photo 1). The postoperative analgesic requirement, postoperative wound pain, time to unassisted ambulation and the pneumoperitoneum height measured on chest X-ray which was taken $24 \mathrm{~h}$ postoperatively were the secondary outcomes of the present study.

\section{Statistical analysis}

All statistical analyses were carried out with IBM SPSS 20.0 software (SPSS Inc., Armonk, NY, USA).

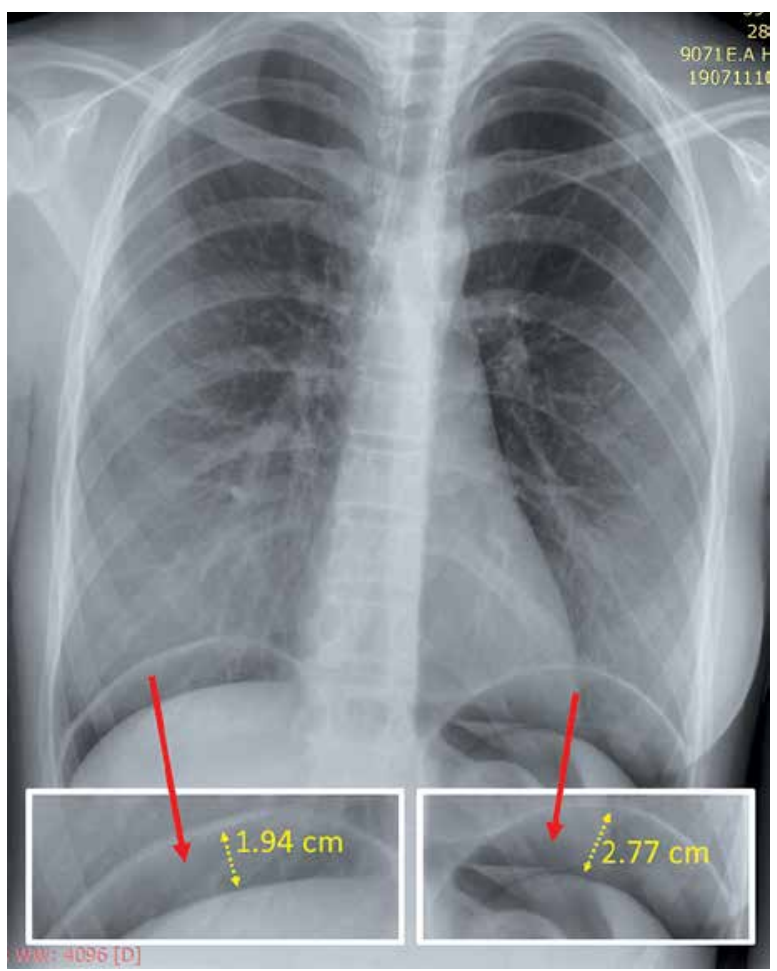

Photo 1. Measurement of pneumoperitoneum on PA chest radiography 
Continuous variables are expressed as mean \pm standard deviation (SD) and categorical variables as number and percentage. The Kolmogorov-Smirnov test was used to determine whether the data were distributed normally. Student's $t$-test was employed for group comparisons and the $\chi^{2}$ test for comparison of dichotomous variables. A $p$-value smaller than 0.05 was accepted as statistically significant.

\section{Results}

The mean age of the study group was $36.4 \pm 11$ years. Thirty-five patients were allocated to receive the PRM at a pressure of $30-40 \mathrm{~cm} \mathrm{H}_{2} \mathrm{O}$ and 37 patients were allocated to receive the PRM at a pressure of $15 \mathrm{~cm} \mathrm{H}_{2} \mathrm{O}$. Patient characteristics are given in Table I. The two groups were similar with regard to age, body mass index, menopause rate laboratory measurements, and operation duration and recovery time.

There were no significant differences in wound pain score or PLSP score between patients receiving the PRM at $30-40 \mathrm{~cm} \mathrm{H}_{2} \mathrm{O}$ and $15 \mathrm{~cm} \mathrm{H}_{2} \mathrm{O}$ during postoperative pain monitoring (Table II). The number of patients receiving rescue analgesics was also not different between the two groups (23\% vs. $11 \%$, $p=0.338)$. The number of subjects receiving intravenous analgesics was similar in the two groups $(23 \%$ vs. $19 \%, p=0.681)$. In addition, there were no significant differences in mean analgesic doses of the two groups (78 $\pm 38 \mathrm{mg}$ vs. $68 \pm 25 \mathrm{mg}, p=0.573$ ).

Table III shows the secondary outcomes. The groups were similar with respect to ambulation time $(13.9 \pm 1.3 \mathrm{~h}$ vs. $13.3 \pm 1.7 \mathrm{~h}, p=0.215)$, length of hospital stay $(1.7 \pm 0.4$ days vs. $1.6 \pm 0.5$ days, $p=0.556)$ and height of the pneumoperitoneum measured on chest X-ray (3.4 $\pm 0.7 \mathrm{~mm}$ vs. $3.2 \pm 0.6 \mathrm{~mm}, p=0.151)$.

\section{Discussion}

The present study investigated whether a low-pressure PRM would exert a similar impact to a moderate-pressure PRM on postoperative shoulder pain and height of pneumoperitoneum on chest $\mathrm{X}$-ray in patients undergoing LS for non-malignant

Table I. Baseline characteristics

\begin{tabular}{|lccc|}
\hline Parameter & $\begin{array}{c}\text { PRM with } 30-40 \mathrm{~cm} \mathrm{H}_{2} \mathrm{O} \\
(n=35)\end{array}$ & $\begin{array}{c}\text { PRM with } 15 \mathrm{~cm} \mathrm{H} \mathrm{H}_{2} \mathrm{O} \\
(n=37)\end{array}$ & $P$-value \\
\hline Age [years] & $37.1 \pm 14.9$ & $35.8 \pm 10.7$ & 0.698 \\
\hline BMI [kg/m²] & $30.1 \pm 4.1$ & $30.5 \pm 3.5$ & 0.624 \\
\hline Menopause (\%) & 16.6 & 20 & 0.473 \\
\hline Operation time [min] & $110.9 \pm 20.2$ & $108.5 \pm 14.7$ & 0.705 \\
\hline Recovery time [min] & $33.4 \pm 4.2$ & $32.2 \pm 5.1$ & 0.321 \\
\hline
\end{tabular}

Data are presented as mean \pm standard deviation. BMI - body mass index; PRM - pulmonary recruitment maneuver.

Table II. Visual analogue scale (VAS) scores for postoperative wound and shoulder pain

\begin{tabular}{|lccc|}
\hline Variables & $\begin{array}{c}\text { PRM with 30-40 } \\
(n=35)\end{array}$ & $\begin{array}{c}\text { PRM } \mathrm{H}_{2} \mathrm{O} \text { with } 15 \mathrm{~cm} \mathrm{H}_{2} \mathrm{O} \\
(n=37)\end{array}$ & $P$-value \\
\hline WP at $6 \mathrm{~h}$ & $5.1 \pm 0.8$ & $5.3 \pm 0.7$ & 0.263 \\
\hline WP at $12 \mathrm{~h}$ & $3.8 \pm 0.6$ & $4.1 \pm 0.8$ & 0.277 \\
\hline WP at $24 \mathrm{~h}$ & $3.1 \pm 0.5$ & $3.2 \pm 0.6$ & 0.380 \\
\hline PLSP at $6 \mathrm{~h}$ & $5.6 \pm 0.9$ & $5.4 \pm 0.8$ & 0.220 \\
\hline PLSP at $12 \mathrm{~h}$ & $4.4 \pm 0.8$ & $4.2 \pm 0.6$ & 0.298 \\
\hline PLSP at 24 $\mathrm{h}$ & $3.3 \pm 0.8$ & $3.1 \pm 0.8$ & 0.218 \\
\hline IV analgesic (dexketoprofen), $n$ & $8(23 \%)$ & $7(19 \%)$ & 0.681 \\
\hline Intravenous analgesic dose [mg] & $78 \pm 38$ & $68 \pm 25$ & 0.573 \\
\hline
\end{tabular}

Data are presented as mean \pm standard deviation. PLSP - post-laparoscopic shoulder pain, PRM - pulmonary recruitment maneuver, WP - wound pain. 
Table III. Secondary outcomes

\begin{tabular}{|lccc|}
\hline Parameter & $\begin{array}{c}\text { PRM with 30-40 } \\
(n=35)\end{array}$ & $\begin{array}{c}\text { PRM } \mathrm{H}_{2} \mathrm{O} \text { with 15 } \mathrm{cm} \mathrm{H}_{2} \mathrm{O} \\
(n=37)\end{array}$ & $P$-value \\
\hline PRRA, $n(\%)$ & $7(23)$ & $4(11)$ & 0.338 \\
\hline Time to ambulation [h] & $13.9 \pm 1.3$ & $13.3 \pm 1.7$ & 0.215 \\
\hline Pneumoperitoneum [mm] & $3.4 \pm 0.7$ & $3.2 \pm 0.6$ & 0.151 \\
\hline LOS [days] & $1.7 \pm 0.4$ & $1.6 \pm 0.5$ & 0.556 \\
\hline
\end{tabular}

Data are presented as mean \pm standard deviation. LOS - length of hospital stay, PRM - pulmonary recruitment maneuver, PRRA - patients receiving rescue analgesics.

causes. Our findings indicate that the PRM with $15 \mathrm{~cm} \mathrm{H} \mathrm{H}_{2} \mathrm{O}$ pressure provides similar efficacy as the PRM with 30 to $40 \mathrm{~cm} \mathrm{H} \mathrm{H}_{2} \mathrm{O}$ with respect to PLSP, wound pain, height of pneumoperitoneum, time of ambulation and length of hospital stay.

Laparoscopic surgery and minimally invasive techniques have been attractive for professionals dealing with gynecologic diseases as these procedures have been shown to reduce surgery-related trauma and postoperative pain, and shorten hospital stay [6]. The laparoscopic technique enhances the recovery process by decreasing perioperative stress and shortening the time required for ambulation and proper gastrointestinal functioning [7]. Compared to conventional open surgical procedures LS enables a more pleasing postoperative course. Spinal anesthesia has been shown to result in less pain compared to general anesthesia in patients undergoing laparoscopic appendectomy [8]. Since one of the most critical advantages of LS is early ambulation, recent evidence indicates that mechanical or pharmacological thromboprophylaxis for prevention of venous thromboembolism is unnecessary in patients undergoing LS for benign causes [9].

Besides the advantages obtained with LS which are mentioned above, some adverse events including PLSP due to the pneumoperitoneum required to distend the abdomen might also occur. Previous data indicate that PLSP might develop in up to $80 \%$ of the subjects undergoing LS within $72 \mathrm{~h}$ of the surgery [1]. Although the underlying mechanism leading to shoulder pain after LS has not been clearly illuminated yet, distension-induced neuropraxia of the phrenic nerve, acidic intraperitoneal medium and remaining abdominal gas are traditionally considered as the major causes of PLSP [10]. The relation of the residual gas volume and the severity of shoulder tip pain was first demonstrated by Jackson et al. where the authors measured the length of arc and height of the gas bubble under each hemi-diaphragm to produce an estimate of the bubble volume [11]. The authors revealed that both length and the height of the subdiaphragmatic gas measured on chest X-rays taken before discharge were significantly correlated with the PLSP score. Supporting the role of residual pneumoperitoneum, the study conducted by Sabzi Sarvestani et al. showed that the amount of the residual pneumoperitoneum was directly associated with the intensity of PLSP in subjects scheduled for laparoscopic cholecystectomy [12].

Intra-abdominal saline injection, sub-diaphragmatic drain insertion and administration of intraperitoneal local anesthetic agents have been studied and exhibited some degree of benefit in the prevention and management of PLSP [13-15]. However, the results were conflicting with the same techniques in further studies and the majority of these methods were impractical for adoption in daily use due to the additional costs and potential adverse effects. Therefore, interventions which aim to reduce the severity of PLSP through the reduction of the remaining gas volume and the decrease in the resultant phrenic nerve stimulation have gained popularity. Among them, the PRM has been the subject of a considerable amount of research. With the application of the PRM, a downwards shift of the diaphragm occurs due to the increased intrathoracic pressure. The change in the position of the diaphragm leads to an increase in the intra-abdominal pressure and thus facilitates the removal of the remaining abdominal gas. The PRM is traditionally performed using maximal inspiratory pressures of $40 \mathrm{~cm} \mathrm{H}_{2} \mathrm{O}$ to $60 \mathrm{~cm} \mathrm{H}_{2} \mathrm{O}$ [16]. The combination of PRM with Trendelenburg positioning was shown to reduce the severity of PLSP compared to controls receiving conventional care in women undergoing laparoscopic surgery for malignant or premalignant gynecological lesions [17]. Neverthe- 
less, there are limited data comparing the low- and high-pressure PRM with respect to their impact on PLSP. Ryu et al. demonstrated that a moderate-pressure PRM using maximal inspiratory pressures of $40 \mathrm{~cm} \mathrm{H}_{2} \mathrm{O}$ was as efficient as a high-pressure PRM with $60 \mathrm{~cm} \mathrm{H} \mathrm{H}_{2} \mathrm{O}$ for removal of the abdominal gas and relief of the PLSP in patients undergoing gynecologic laparoscopy [18]. Recently, Lee et al. investigated the impact of PRM with a maximal inspiratory pressure of $30 \mathrm{~cm} \mathrm{H}_{2} \mathrm{O}$ on PLSP in patients who were scheduled for elective gynecologic laparoscopy [19]. Through the significant difference between the PRM group and the controls, the authors reached a conclusion that performing the PRM with $30 \mathrm{~cm} \mathrm{H}_{2} \mathrm{O}$ was beneficial in reducing PLSP. However, whether a low-pressure PRM with a maximal inspiratory pressure of $15 \mathrm{~cm} \mathrm{H}_{2} \mathrm{O}$ provides additional benefit in the management of PLSP is still not clear.

Our findings demonstrated that a low-pressure PRM with a maximal inspiratory pressure of $15 \mathrm{~cm}$ $\mathrm{H}_{2} \mathrm{O}$ is as effective as a moderate-pressure PRM performed using 30 to $40 \mathrm{~cm} \mathrm{H}_{2} \mathrm{O}$ pressures. Our results also revealed that a low-pressure PRM is not inferior to a moderate-pressure PRM in terms of time to ambulation, the height of the pneumoperitoneum measured on chest X-ray and the length of hospital stay.

With this background in mind, we suggest that the implementation of a low-pressure PRM is an effective method for preventing shoulder tip pain and wound pain developing after LS. We also suppose that a low-pressure PRM might eliminate the adverse events that might potentially occur with higher pressure PRM. As shown previously, when higher pressures were used for the PRM, several complications including barotrauma and hemodynamic deterioration may occur [3, 4, 20, 21]. However, the PRM with a constant driving pressure of $15 \mathrm{~cm} \mathrm{H}_{2} \mathrm{O}$ was tolerated well and improved oxygenation in an animal model under general anesthesia [22]. Since the optimal inspiratory pressure used for PRM to prevent PLSP has not been established well, given the similar efficacy of the low-pressure PRM and moderate-pressure PRM, we suggest that lower maximal inspiratory pressure of $15 \mathrm{~cm} \mathrm{H}_{2} \mathrm{O}$ might be preferred to avoid the potential complications of PRM with higher pressures.

There are some limitations to be mentioned concerning the present study. We enrolled patients scheduled for gynecologic procedures; therefore, one may feel troubled whether or not our findings could be projected to other LS procedures. Nonetheless, the driving pathophysiological mechanism for PLSP is similar in all LS procedures. Thus, the beneficial effects of the low-pressure PRM might be generalized to all kinds of LS. Second, we suppose that a low-pressure PRM might prevent potential alveolar barotrauma and hemodynamic adverse events which might occur with the moderate or higher pressure PRM. The lack of data concerning alveolar damage or the hemodynamic response in both groups is another limitation of this study. Additionally, patient monitoring for PLSP was maintained up to $24 \mathrm{~h}$ in our study. However, PLSP may persist until $72 \mathrm{~h}$ of surgery. Finally, although recent studies showed favorable results with deep neuromuscular blockade in laparoscopic surgery, moderate neuromuscular blockade was used in our study [23]. Further studies focusing on pain intensity and level of neuromuscular blockade are required in patients undergoing LS.

\section{Conclusions}

The present study clearly demonstrates that a low-pressure PRM with a maximal inspiratory pressure of $15 \mathrm{~cm} \mathrm{H}_{2} \mathrm{O}$ pressure provides similar benefit when compared to a moderate-pressure PRM with a maximal inspiratory pressure of $30-40 \mathrm{~cm} \mathrm{H}_{2} \mathrm{O}$ in patients undergoing gynecologic LS. A low-pressure PRM also appears equal to a moderate-pressure PRM with regard to the height of the pneumoperitoneum measured on chest X-ray. We suggest that even a low-pressure PRM is sufficient to evacuate the remaining abdominal gas following LS. Given the similar efficacy of low-pressure PRM and moderate-pressure PRM in preventing PLSP, we suggest that a lower maximal inspiratory pressure of $15 \mathrm{~cm}$ $\mathrm{H}_{2} \mathrm{O}$ might be preferred to avoid the potential complications of the PRM with higher pressures.

Trial registration number: ClinicalTrials.gov (NCT03970473).

\section{Conflict of interest}

The authors declare no conflict of interest.

\section{References}

1. Phelps P, Cakmakkaya OS, Apfel CC, Radke OC. A simple clinical maneuver to reduce laparoscopy-induced shoulder pain: a randomized controlled trial. Obstet Gynecol 2008; 111: 1155-60.

2. Kiyak H, Yilmaz G, Ay N. Semi-Fowler positioning in addition to the pulmonary recruitment manoeuvre reduces shoulder pain 
following gynaecologic laparoscopic surgery. Videosurgery Miniinv DOI: https://doi.org/10.5114/wiitm.2019.84384.

3. Garcia-Fernandez J, Canfran S, de Segura IA, et al. Pressure safety range of barotrauma with lung recruitment manoeuvres: a randomised experimental study in a healthy animal model. Eur J Anaesthesiol 2013; 30: 567-74.

4. Lovas A, Szakmany T. Haemodynamic effects of lung recruitment manoeuvres. Biomed Res Int 2015; 2015: 478970.

5. Faul F, Erdfelder E, Buchner A, Lang AG. Statistical power analyses using $G^{*}$ Power 3.1: tests for correlation and regression analyses. Behav Res Methods 2009; 41: 1149-60.

6. Pache B, Joliat GR, Hubner M et al. Cost-analysis of enhanced recovery after surgery (ERAS) program in gynecologic surgery. Gynecol Oncol 2019; 154: 388-93.

7. Yilmaz G, Akca A, Aydin N. Enhanced recovery after surgery (ERAS) versus conventional postoperative care in patients undergoing abdominal hysterectomies. Ginekol Pol 2018; 89: 351-6.

8. Erdem VM, Donmez T, Uzman S et al. Spinal/epidural block as an alternative to general anesthesia for laparoscopic appendectomy: a prospective randomized clinical study. Videosurgery Miniinv 2018; 13: 148-56.

9. Sturlese E, Triolo O, Grasso R, et al. Thromboembolism prophylaxis in laparoscopic surgery for gynecologic benign diseases. Results of a single center experience in 922 procedures. Ann Ital Chir 2017; 88: 342-7.

10. Abbott J, Hawe J, Srivastava P, et al. Intraperitoneal gas drain to reduce pain after laparoscopy: randomized masked trial. Obstet Gynecol 2001; 98: 97-100.

11. Jackson SA, Laurence AS, Hill JC. Does post-laparoscopy pain relate to residual carbon dioxide? Anaesthesia 1996; 51: 485-7.

12. Sabzi Sarvestani A, Zamiri M. Residual pneumoperitoneum volume and postlaparoscopic cholecystectomy pain. Anesth Pain Med 2014; 4: e17366

13. Lepner U, Goroshina J, Samarutel J. Postoperative pain relief after laparoscopic cholecystectomy: a randomised prospective double-blind clinical trial. Scand J Surg 2003; 92: 121-4.

14. Donatsky AM, Bjerrum F, Gogenur I. Intraperitoneal instillation of saline and local anesthesia for prevention of shoulder pain after laparoscopic cholecystectomy: a systematic review. Surg Endosc 2013; 27: 2283-92.

15. Donatsky AM, Bjerrum F, Gogenur I. Surgical techniques to minimize shoulder pain after laparoscopic cholecystectomy. A systematic review. Surg Endosc 2013; 27: 2275-82.

16. Pergialiotis V, Vlachos DE, Kontzoglou K, et al. Pulmonary recruitment maneuver to reduce pain after laparoscopy: a metaanalysis of randomized controlled trials. Surg Endosc 2015; 29: 2101-8.

17. Gungorduk K, Asicioglu O, Ozdemir IA. Effect of the pulmonary recruitment maneuver on pain after laparoscopic gynecological oncologic surgery: a prospective randomized trial. J Gynecol Oncol 2018; 29: e92.

18. Ryu K, Choi W, Shim J, Song T. The impact of a pulmonary recruitment maneuver to reduce post-laparoscopic shoulder pain: a randomized controlled trial. Eur J Obstet Gynecol Reprod Biol 2017; 208: 55-60.

19. Lee J, Park C, Kim J, et al. Effect of low-pressure pulmonary recruitment maneuver on postlaparoscopic shoulder pain: randomized controlled trial. J Minim Invasive Gynecol doi: 10.1016/j.jmig.2019.03.020.

20. da Silva PS, de Aguiar VE, Fonseca MC. Iatrogenic pneumothorax in mechanically ventilated children: Incidence, risk factors and other outcomes. Heart Lung 2015; 44: 238-42.

21. Hsu CW, Sun SF. latrogenic pneumothorax related to mechanical ventilation. World J Crit Care Med 2014; 3: 8-14.

22. de la Osa AM, Garcia-Fernandez J, Llorente-Cantarero FJ, et al. Lung recruitment manoeuvres do not cause haemodynamic instability or oxidative stress but improve oxygenation and lung mechanics in a newborn animal model: an observational study. Eur J Anaesthesiol 2014; 31: 457-65.

23. Park SK, Son YG, Yoo S, et al. Deep vs. moderate neuromuscular blockade during laparoscopic surgery: a systematic review and meta-analysis. Eur J Anaesthesiol 2018; 35: 867-75.

Received: 26.08.2019, accepted: 25.10.2019. 